\title{
Experimental results for an experimental condensation heat exchanger with a spiral minichanel tube. Comparison to numerical simulations
}

\author{
P. Peukert ${ }^{1}$, J. Hrubý ${ }^{1}$ \\ ${ }^{1}$ Institute of Thermomechanics AS CR, v. v. i., department of Thermodynamics, Dolejškova 1402/5, 18200 Prague 8, Czech \\ Republic
}

\begin{abstract}
The paper describes new results for an experimental heat exchanger equipped with a single corrugated capillary tube, basic information about the measurements and the experimental setup. Some of the results were compared with numerical simulations.
\end{abstract}

\section{Introduction}

Heat transfer phenomena are present in various technical applications: it ranges from simple things like boiling water in a kettle over managing temperature conditions inside buildings to sophisticated systems for energy extraction from different heat sources. In the mentioned applications, heat exchangers are used for heat transfer between two media. In common home heating areas, the heat is taken from gaseous combustion products and transferred to water which is later distributed over the heated building. Today, also a very popular field is making use of the thermal waste energy from various technologies for water and building heating. For companies it is a reliable, functional, cheap, small, maintenance-free, and, therefore, popular solution.

A special kind of heat exchangers, are the condensation heat exchangers. In figure 2 , an industrial condensation heat exchanger is shown, which was installed instead of an older broken one which used waste steam from a porcelain production plant. The new heat exchanger demands less area, is cheaper and has no maintenance costs for few years.

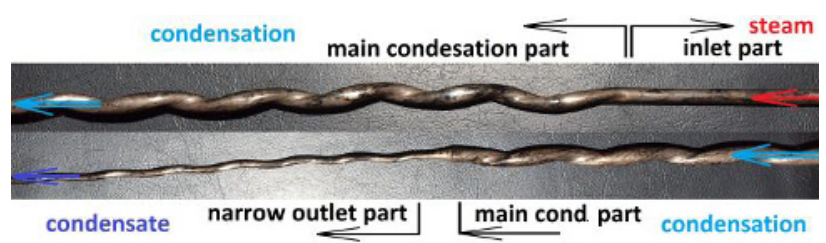

Fig. 1. The condensation minichannel tube. Left side down.

The mentioned condensation heat exchanger contains a bundle of miniature corrugated tubes in which the steam condensation occurs and outside flows the heated water. A part of such a tube is depictured in figure 1. According to Garimela [5], it could be called as micro or more accurate as minichannel tube. Various effects which take place inside the tube have different weights than for typical macroscopic cases. For example, buoyancy forces were here less prominent in such cases than viscous shear and surface tension forces. The heat transfer in relation to the geometry of the corrugated mini-channel tubes is still not examined adequately. For this purpose a simplified experimental heat exchanger was constructed. It should allow a better investigation of the processes inside the heat exchanger and provide data enabling further improvement of the performance of the condensation heat exchangers.

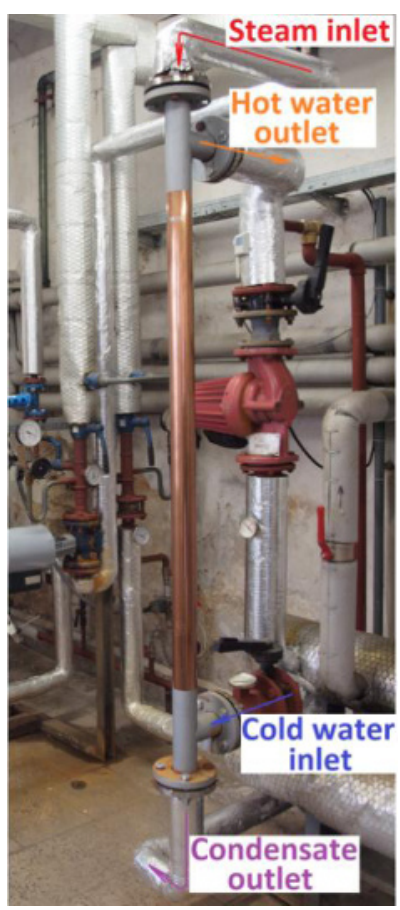

Fig. 2. Connected industrial heat exchanger 


\section{Experimental setup}

The experimental setup could be divided in two major parts. The first part is the steam generating system. It is a unique system [1] working as a supply system for various research applications.

The second part is the experimental heat exchanger with its accessories. Its simplified scheme is shown in figure 2 . The slightly overheated steam enters the heat exchanger from the top. The temperature of the inlet steam with a T-type thermocouple (the same type is used also at other locations) and its pressure is measured with a pressure sensor Druck PMP 1400 with range of 15 bara. The condensation process occurs during the flow through the spiral minichanel tube. Another thermocouple is located at the outlet to determine the temperature of the condensate. The regulation of the flow rate is provided by a control valve at the rotameter Omega FL-3804ST. The steam pressure is controlled using the steam generating system. The purpose of the rotameter is measuring the approximate value of the flow rate. For the computations, a more accurate weighing method used employing an accurate scale and a stopwatch. With this measured quantities, the specific enthalpy at the inlet $h_{\mathrm{s} \text {,in }}$ and

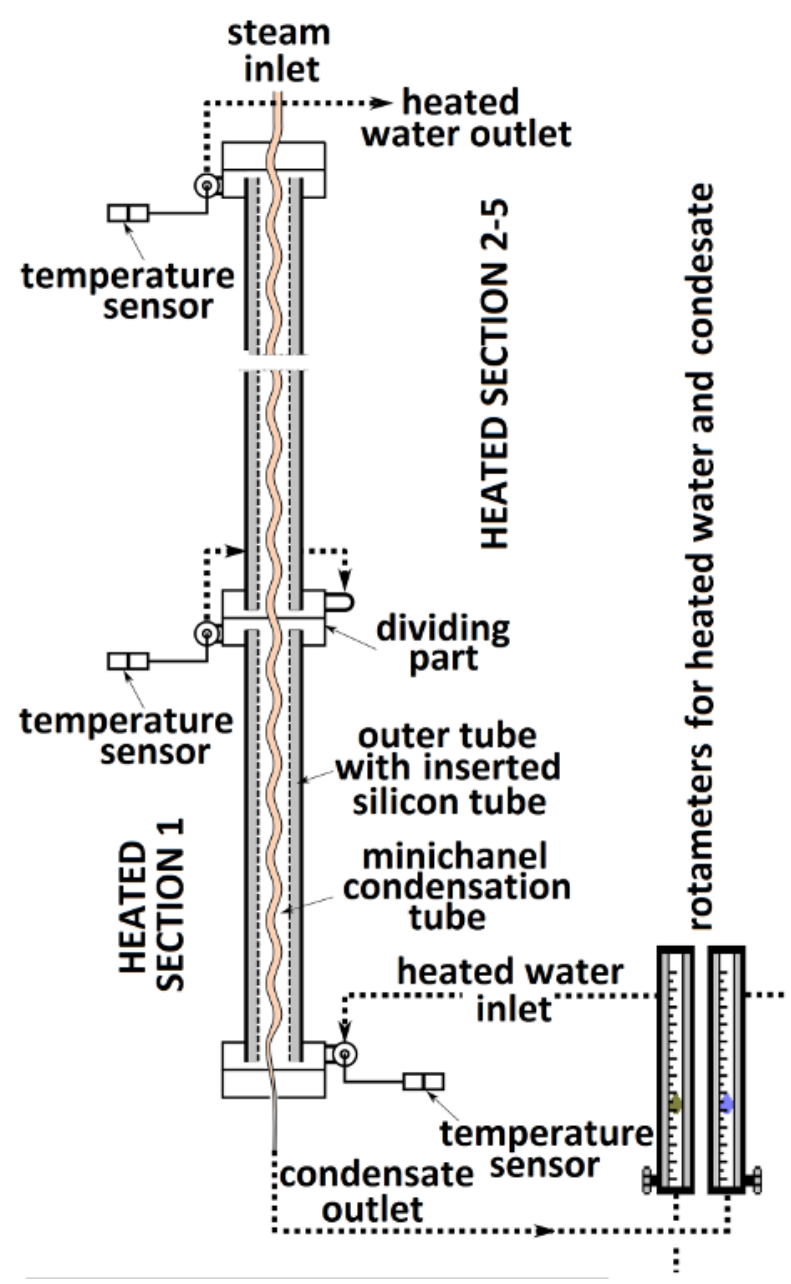

Fig. 3. Scheme of the experimental heat exchanger (only two sections shown, in reality 5 sections) outlet $h_{\mathrm{c} \text {,out }}$ could be determined using the so-called industrial formulation of thermodynamic properties of water and steam IAPWS-IF97 [2]. The total heat transfer rate from the condensing medium to the heated water was obtained as

$$
\dot{Q}_{\text {total }}=\dot{m}_{\mathrm{c}}\left(h_{\mathrm{c}, \text { out }}-h_{\mathrm{s}, \text { in }}\right)
$$

where $\dot{m}_{\mathrm{c}}$ is the condensate/steam mass flow rate.

From the bottom side, water flows trough the second rotameter (Omega FL-3840C) equipped with a control valve. Because of higher flow rates and a relative constant temperature at the inlet, this method of the flow rate measurement was sufficiently accurate. The heat exchanger had a counterflow arrangement. To determine the spatial distribution of the heat transfer along the capillary tube, the outer tube was divided in five sections. The sections are not equidistant because from preliminary results it was clear that the condensation for most of the measured cases occurs in the first (upper) part of the minichanel tube. Therefore, was chosen to divide the upper two sections equally and insert there dividing parts. The three sections on the bottom, where near no condensation had appeared, were merged to one. At the inlet, outlet and every diving part is measured again the temperature to gather datas for compution of specific entalpies at the given position. So it is posible to compute heat transfer to the heated water in every section:

$$
\dot{Q}_{i}=\dot{m}_{l}\left(h_{i+1}-h_{i}\right)
$$

Here $i=1, \ldots, 5$ represents the number of the section starting from the bottom. The enthalpies correspond to temperatures measured at the heated water enrance $(i=1)$, between the sections $(i=2, \ldots, 5)$ and at the exit of the heated water. $\dot{m}_{l}$ is the mass flow rate of heated water in the anular channel.

All measured data were collected via the data acquisition unit HP 34970A (today Agilent) direct to a computer. The scale data from both rotameters and weight and time values from were added manually. All the values were evaluated with an in-house writen algorithm in the Matlab ${ }^{\circledR}$ working environment. This approach was chosen for easy data handling. The amount of data exceeds 1500 measurements.

\section{Experimental results}

The new experimental results given in this section correspond to the configuration where the outer tube inner diameter is $10 \mathrm{~mm}$ and the corrugated minichannel tube had an outer diameter of $4 \mathrm{~mm}$. The center of the circular cross-section of the capillary tube is displaced $2 \mathrm{~mm}$ from the axis of the outer tube and it rotates in a helical manner with a pitch of $15 \mathrm{~mm}$. The hydraulic diameter for this case is $6 \mathrm{~mm}$. Previously, Peukert and Hrubý [3] provided data for a hydraulic diameter of $12 \mathrm{~mm}$. 
Table 1 presents three correlations between scale values of the rotameters and the approximate flow rate to give the reader an approximate knowledge of the present flow rates inside the experimental heat exchanger and ensure a good interpretation of the results.

Table 1. Calibration data of rotameters

\begin{tabular}{|c||c|c|}
\hline \multirow{2}{*}{\begin{tabular}{c}
\multirow{2}{*}{$\begin{array}{c}\text { rotameter } \\
\text { reading }\end{array}$} \\
\cline { 2 - 3 }
\end{tabular}} & $\begin{array}{c}\text { Volumetric flow rate } \\
\text { determined by weighing }\end{array}$ \\
\cline { 2 - 3 }$\left[\begin{array}{c}\text { Condensate } \\
@ 22^{\circ} \mathrm{C}\end{array}\right.$ & $\begin{array}{c}\text { Heated water } \\
@ 18^{\circ} \mathrm{C}\end{array}$ \\
\hline 50 & {$[\mathrm{ml} / \mathrm{min}]$} & {$[\mathrm{ml} / \mathrm{min}]$} \\
\hline 100 & 50 & 550 \\
\hline 150 & 98 & 1246 \\
\hline
\end{tabular}

In figures 4 to 6 show the mean fluxes for each section for various experimental cases. The figures also give the readings of the rotameters.

Figure 4 presents three cases, which are representative for different steam/condensate flow rates and approximately the same inlet pressure of 4 bar ( \pm 0.06 bar) and temperature $150{ }^{\circ} \mathrm{C}$ and constant heated water flow rate at the rotameter scale value of $150 \mathrm{~mm}$. It can be observed that the heat transfer rate grows with the increase of the steam flow rate. It is clear that the total heat transfer rates increase proportional with the flow rate up to a limit. The given picture gives an overview about the heat transfer distribution among the given sections. For condensate flow rate of $40 \mathrm{~mm}$, the condensation starts immediately at section 5 and ends within section 3 . For the second case of heated water flow rate of $100 \mathrm{~mm}$ the condensation starts in section 5 , reaches its maximum in section 4 , decreases slightly in section 3 and ends at section 2 . In the last case, the condensation starts again in section 5 but a relative long part of convectional heat transfer from overheated steam is present, later the intensity of condensation increase and reach its maximum

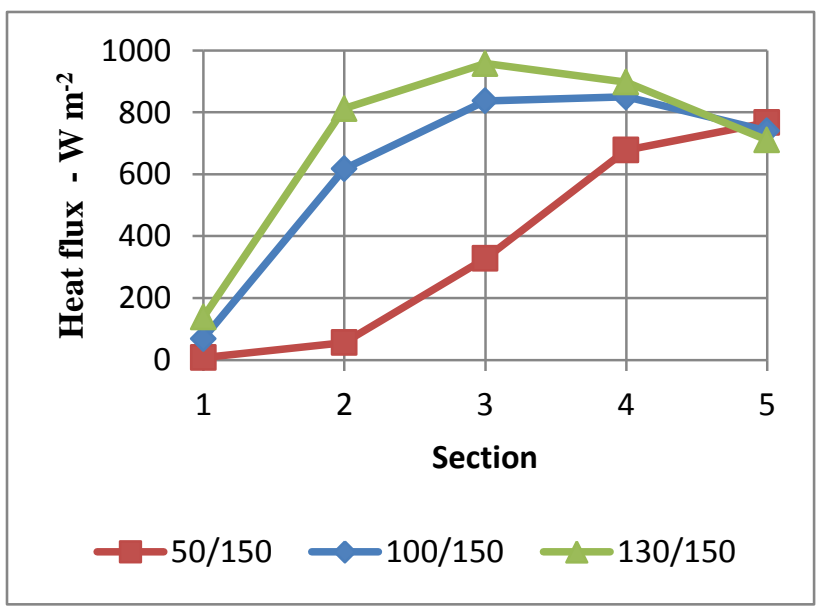

Fig. 4. Heat flux for given sections at 4 bar pressure inside the minichanel tube tube.

at section 3. Within section 2 the condensation ends and further single-phase heat transfer from the condensed water occurs. The case $100 / 150$ is used later for the numerical simulation for convective heat transfer from the inner tubes outer wall to the heated water bulk.

Figure 5 shows a chart of influence of heated water flow rate on the heat transfer distribution along sections for cases where the scale flow rate of $50 \mathrm{~mm}$ is around its support maximum for the inlet pressure of 2.3 bar. For these cases it can be observed that the heat transfer rate distribution strongly depends on the heated water flow rate. This effect could show such dependence, because there is a high chance that the flow regime changes from laminar to turbulent. Therefore, the minichannel wall subcooling is higher and that means also a higher gradient between the inner wall and the steam.

For the first case where the rotameter condensate flow rate is $40 \mathrm{~mm}$ the condensation occurs in all sections and increase downward. But in section five is mostly the overheated steam cools down to the saturation. The increase of the heat transfer downwards can be explained with the fact that near over the whole length the steam condenses and the tube wall has approximately constant temperature, but due the counterflow configuration, the temperature difference between the saturation temperature and the actual heated water temperature at the given location decreases downwards. In favor of this explanation, the fact speaks that the temperature of the condensate at the outlet of the minichanel tube is near $80{ }^{\circ} \mathrm{C}$ and on the other side the outlet at the upper part of the heated water is $92{ }^{\circ} \mathrm{C}$. This evolution changes rapidly if the heated water flow rate is increased. The main part of the heat transfer slowly moves to the upper part. But also for the highest heated water flow rates it is not possible to reach that the highest flow heat transfer rate moves to the $5^{\text {th }}$ section. Only it could be observed that in cases with higher flow rates than $90 \mathrm{~mm}$ of heated water the condensation stopped probably at section 2 .

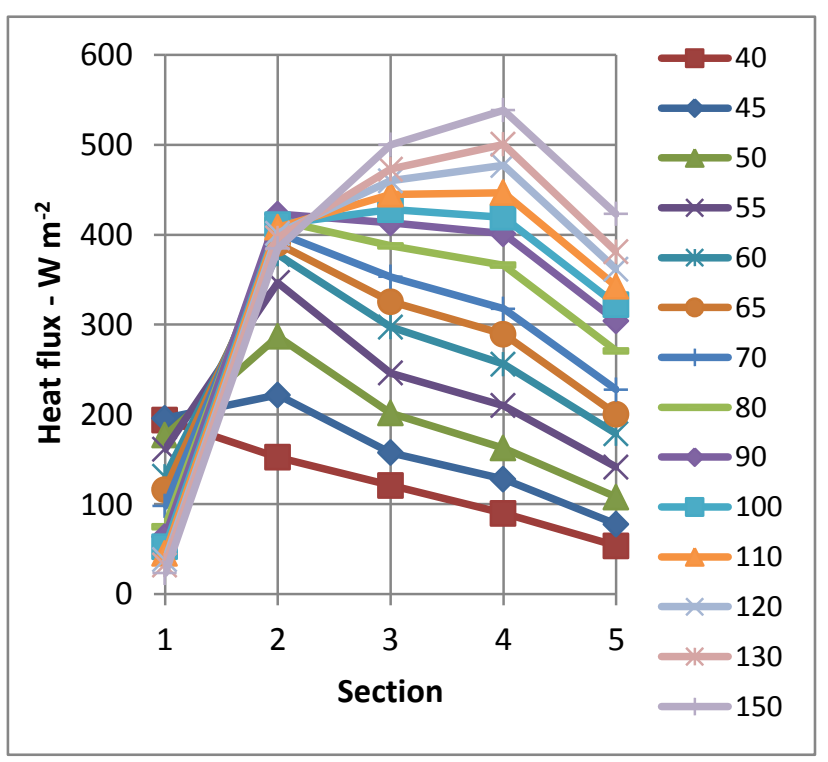

Fig. 5. Heat flux for given sections at a pressure of 2.3 bar inside the minichanel spiral tube and a condensate scale value flow rate of $50 \mathrm{~mm}$.

Another interesting step is the comparison of the previous case with figure 6 . The pressure was increased to 6.3 bar 
and while the condensate flow rates and most of the heated water flow rates remain the same as for cases shown in figure 5. For both cases the inlet temperature was approximately $10{ }^{\circ} \mathrm{C}$ above the saturation. It is clear that the energy potential available in the second case is higher due the higher pressure and temperature of the inlet steam. The higher saturation temperature means also a higher temperature at the condensation part and also the wall so that the convection on the outer surface of the minichannel to the heated water is more intensive. For 6.3 bar the water steam saturation temperature approximately was 160 compared to 125 Celsius degrees for 2.3 bar. This is approximately an increase of $30 \%$ in the temperature difference, 140 vs. 105 Celsius degrees. At a first look it is clear that for the same heated water flow rate for both cases the second one is more than two times higher. The condensation process had ended already at next-to-last section (4). Like in the previous case, the heat flux increases with the heated water flow rate but it falls in the section 1 to 3 . While in the previous case (figure 5) the heat flux increased approximately two times with increasing heated water flow rate, here was risen only by one third. For the heated water flow rates from 40 to $60 \mathrm{~mm}$ on the rotameter scales, the condensation process is about of the same intensity, but further increasing the heat transfer became more prominent in section 5. That is also clear, because again the temperature difference is rising. For these cases the outlet heated water temperature ranges from $92{ }^{\circ} \mathrm{C}$ for low heated water flow values to $38^{\circ} \mathrm{C}$ for the higher ones.

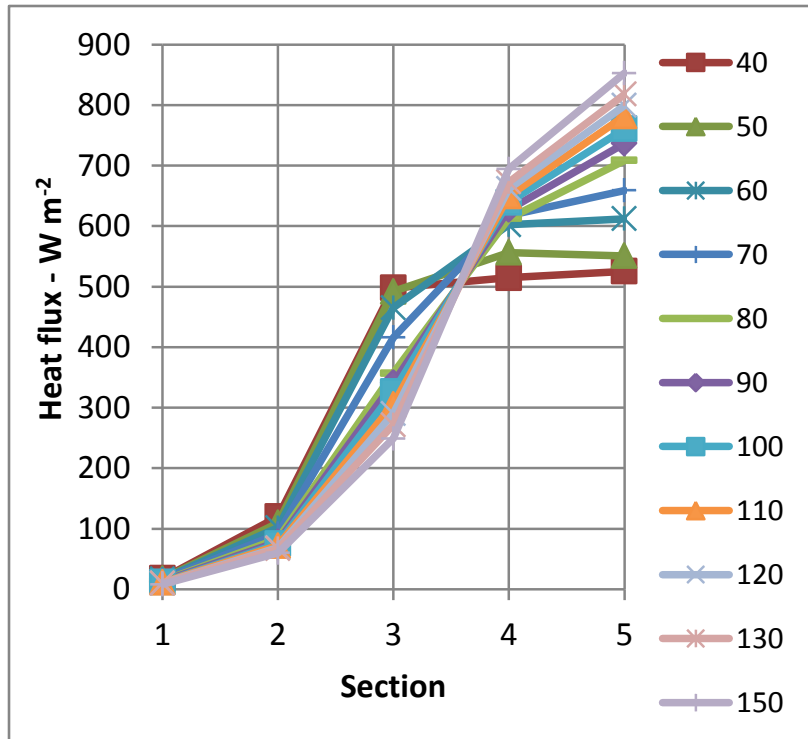

Fig. 6. Heat flux for given sections at pressure of $6.3 \mathrm{bar}$ inside the minichanel spiral tube and a condensate scale value flow rate of $50 \mathrm{~mm}$.

Figures 4 to 6 show only a selection of representative data to give an idea of how the heat transfer distribution along the condensation minichannel corrugated tube looks. From the complete measurement data collection a lot of them showing a potential to increase the heat transfer rates by change the geometrical parameters on the surface of the minichannel tube which is surrounded by the heated water.

\section{Numerical computations}

Because the condensation process in this case is too complex and is not possible to simulate it properly, we choose to perform simulations of the convective heat transfer from the outer surface of the minichanel tube to the heated water. The used software was "Ansys Fluent 14 " $®$ and the A were prepared by Solidworks ${ }^{\circledR}$.

In reality the condensation appears if the steam is somewhat subcooled under the saturation temperature corresponding to the pressure inside the capillary. However, for the cases studied the subcooling is rather small. The thermal conductivity of the tube wall is high (copper), so that a uniform temperature equal to the saturation temperature for given pressure can be assumed at the outer surface of the minichannel tube.

After a careful consideration the case "100/150" shown in figure 4 was chosen for simulation. One of the indicators of the complete and constant condensation process over the whole section is that it appears at same amount in section 3 and 4 so that could be the presumption of the constant wall temperature at the steam saturation parameter

As a check for the simulations we choose the case with an annular flow at the same hydrodynamic diameter and same boundary conditions but the inner surface is cylindrical instead of the spiral one. The values for comparison were here computed by the using relations found in Hewit [4]:

$$
N u_{\mathrm{an}}=N u_{\mathrm{fd}} 0.86\left(\frac{d_{\mathrm{i}}}{d_{\mathrm{o}}}\right) .
$$

Where $d_{\mathrm{i}}-$ is the inner diameter and $d_{\mathrm{o}}-$ is the outer diameter of the annulus. $N u_{\mathrm{fd}}$ is the Nusselt number of a common tube with the same hydraulic radius:

$$
N u_{\mathrm{fd}}=\frac{\frac{f}{8}(R e-1000) P r}{1+12.7 \sqrt{\frac{f}{8}}\left(\operatorname{Pr}^{\frac{2}{3}}-1\right)}\left[1+\frac{d_{\mathrm{ch}}}{L}\right] .
$$

Where $d_{\mathrm{ch}}$ is the diameter of a tube with an equivalent diameter and $f$ is defined:

$$
f=\left[1.82 \log _{10}(R e)-1.64\right]^{-2} .
$$

The range of validity of equation (3) to (5) is from Reynolds number of 2300 to $10^{6}$, that means the region from transient to turbulent regime. The computed case corresponds to a Reynolds number of 2375 so it is just applicable. The result is shown in figure 9.

For all numerical simulations the same boundary conditions were used as presented in figure 7 . The inlet velocity and temperature were set correspond to the experimental data at the inlet of heated water to section 3. The heated wall was set to the saturation temperature in relation to the inlet pressure in the minichannel tube. Zero heat flux was assumed at the inner wall of the outer tube because of negligible loses. The pressure outlet was set to zero overpressure. The difference between the inlet and 
outlet temperature was within ten degrees so the material properties were set to constant values. In figure 8 the cross-section of is shown to help to understand the chosen boundary conditions.

Two types of meshes were used. One had a uniform design, the second had a more sophisticated boundary layer. The mesh was a hybrid one, as based elements were used tetrahedrons the other were hexahedrons.

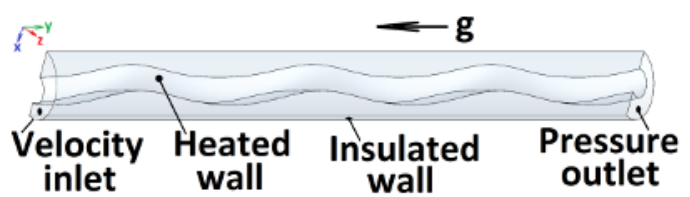

Fig. 7. Sectional view of the model with boundary conditions.

In Fluent ${ }^{\circledR}$ was used a user defined function to get average temperatures for the inlet an outlet surface:

$$
\widetilde{T}=\frac{1}{\dot{m}} \int_{A} \rho U_{j} T \mathrm{~d} A
$$

The result of equation (6) was Where $\mathrm{T}$ is the average temperature, $r$ the density of the fluid, $U_{j}$ the velocity along the channel, $T$ the actual temperature and $\mathrm{d} A-$ the surface diferential.

For heat transfer computation was used equation (7) :

$$
\dot{Q}=\dot{m} c_{p}\left(\frac{1}{\dot{m}} \int_{A \text { in }} \rho U_{j} T \mathrm{~d} A-\frac{1}{\dot{m}} \int_{\text {Aout }} \rho U_{j} T \mathrm{~d} A\right) .
$$

$c_{p}$ is the isobaric heat capacity. For the given range was assumed a constant value of $4180 \mathrm{~J} \mathrm{~kg}^{-1} \mathrm{~K}^{-1}$. For comparison was chosen the heat transfer coefficient:

$$
\alpha=\frac{\dot{Q}}{\left(T_{\mathrm{w}}-T_{1, \mathrm{in}}\right) A_{C}} .
$$

Where $T_{\mathrm{w}}$ is the constant wall temperature, $\mathrm{T}_{1, \text { in }}$ is the constant inlet temperature and $A_{\mathrm{c}}$ is the cylindrical surface of the inner tube.

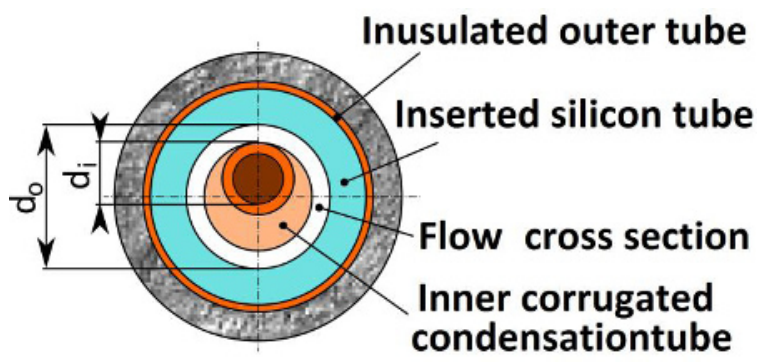

Fig. 8. Heat exchanger cross section normal to the tube axis

To compare the possible simulation methods, a large number of computations with different settings and with different laminar/turbulent flow models. With regard to the inlet Reynolds number and the geometrical shape it was also assumed that some turbulence could occur. For this reason also some turbulent models were used: the komega, k-omega SST (which is a combination of k- omega and k-epsilon model) and Spalart Almaras. Some of the models were also run in the unsteady regime and the results were obtained for times where the flow was stabilized.

\section{Comparison}

A summary of the computations is shown in figure 9, which compares the convective heat transfer coefficient for the different approaches for a straight and a spiral inner tube.

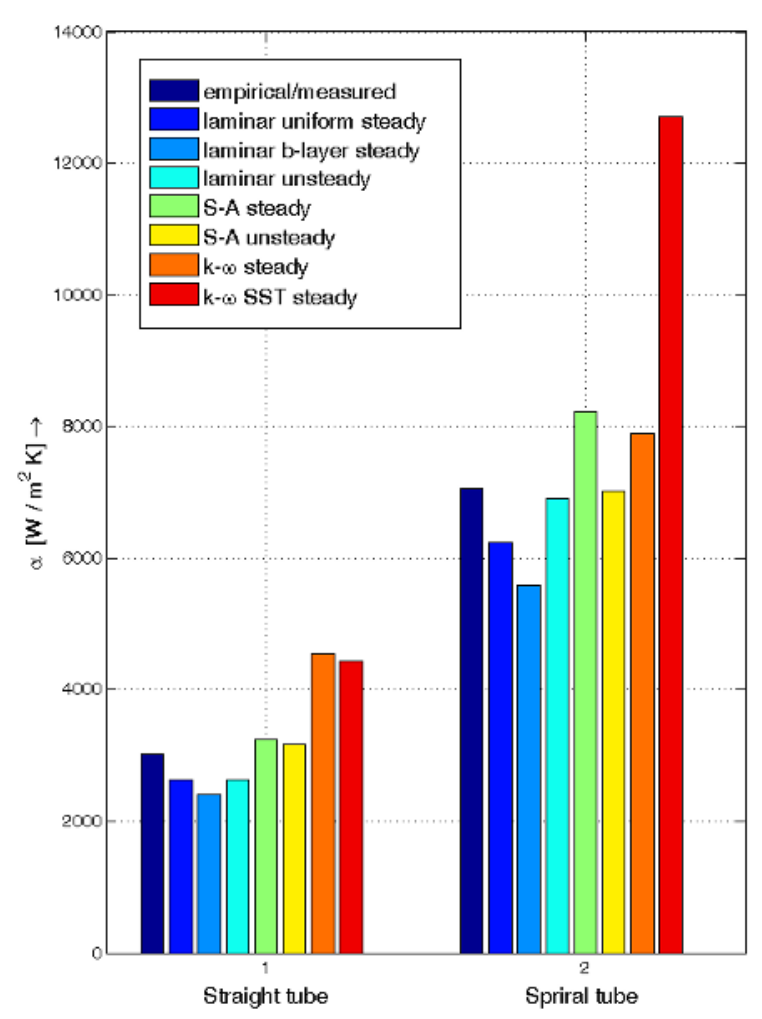

Fig. 9. Comparison of convection coefficient from the inner straight/spiral tube to the annular flow

For the relative low annular flow it appears that the difference between the uniform mesh and the one with a sophisticated boundary layer mesh makes a marginal difference but the computation time for both the spiral and straight tube cases strongly increased.

For the straight tube it appears that the Spalart Almaras model provides a best fit. The reason could lay in its well developed wall functions. However, this model was developed for different applications and therefore should be handled with care. The laminar model also provided a sufficient prediction of the heat transfer coefficient. The turbulent models were here not appropriate, because of the geometrical arrangement and the low Reynolds number indicate that the flow remains in a laminar regime for this case.

A different situation is present for the spiral minichannel tube. Here the results suggest that some turbulent structures could appear. For this reason also could be sufficient the k-omega standard model. The k- 
omega SST which use at wall regions the k-omega standard approach and at wall far regions the k-epsilon approach seems not useful, probably it would more suit for higher Reynolds numbers. The laminar model suits also well especially the unsteady one. The easy explanation is that this case is just at the border between the laminar and turbulent regimes. From the point of view of prediction of the heat transfer, the computation is sufficient. Again the Spalart Almaras model seems also useful, but also here its results should interpreted with care. For a better assesment if applicability of this model to similar cases, a further test and more investigation work needs to be done.

\section{Conclusions}

Further measurements are planned for hydraulic diameters of 8 and $4 \mathrm{~mm}$. While the variant with $4 \mathrm{~mm}$ best fits the real case, it should be taken in account that, in comparison to the real industrial heat exchanger, the heated water is surrounded with further corrugated minichanel tubes while the experimental one it is surrounded by the insulated tube wall.

Also is planned to perform a further evaluation of all measured values to obtain coefficients for empirical computations of heat transfer with this spiral tube. Another goal is the more precise determination of the location of the condensation in the inner tube.
Some further simulations will be done for the heated water. It appears that there is a potential for heat transfer improvement on the heated water side which could help to increase the heat transfer rates. However, further computations are needed for validation especially in cases where transient or turbulent flow occurs.

\section{Acknowledgement}

We gratefully acknowledge the support by Grant No. 101/09/1633 of the Grant Agency of the Czech Republic. The capillary tubes were kindly provided by the company Glazer, Cheb, Czech Republic. We gratefully acknowledge technical assistance by Bohuslav Smid (mechanical design) and Miroslav Pavelka (electrical design).

\section{References}

1. P. Peukert, Diplomová práce (TUL, 2006)

2. Revised Release on the IAPWS Industrial Formulation of Water and Steam (Lucerne, Switzerland, 2007)

3. P. Peukert, J. Hruby, EFM 2009 (TUL, 2009)

4. G. F. Hewit, Heat exchanger design handbook (1998)

5. S. Garimella, S Kandlikar, Heat transfer and fluid flow in minichannels and microchannels (Kidlington, Oxford, UK, Elsevier, 2006) 